\title{
Research on Closed-Loop Supply Chain with the Third Party Recycler Based on Consumer Preference
}

\author{
Jiangbo Zheng, Juan Wu \\ School of Management, Jinan University, Guangzhou, China \\ Email: Annawu2030@163.com
}

Received 17 March 2016; accepted 20 April 2016; published 26 April 2016

Copyright (C) 2016 by authors and Scientific Research Publishing Inc.

This work is licensed under the Creative Commons Attribution International License (CC BY). http://creativecommons.org/licenses/by/4.0/

\begin{abstract}
This paper discusses the closed-loop supply chain which is composed of a manufacturer, a retailer and the third-party recycler, the market dominated by manufacturer. Consumer conveys different preference for new products and remanufactured products. From this base, we segment markets and determine the market demand of different products. Then, we set up a closed-loop supply chain with the third party collecting decision-making model, the problem of different members of the optimal decision is discussed. Finally, the influence of consumer preference on sale price, wholesale price, recovery rate and profits is discussed through a numerical example analysis.
\end{abstract}

Keywords

Consumer Preference, Closed-Loop Supply Chain, Stackelberg Model

\section{Introduction}

With the continuous development of science and technology and the increasing of people's living standard, the product life cycle is shortened, more and more waste products, accompanied by resource crisis and environmental problems are becoming increasingly prominent. Therefore, developing circular economy, maintaining social sustainable development and building a resource saving and environment friendly society have become an urgent strategic issues. Remanufacture engineering is one of the main ways to realize the circulation economy, to alleviate the shortage of resources and sustainable development. Recycling and remanufacturing of waste products has become an important strategic operation mode of modern enterprise, and it can produce significant economic benefit and social benefit [1].

Closed-loop supply chain is a very important aspect of supply chain management, it is a hot topic in recent years. Currently, representative studies on the closed-loop supply mainly include: Zhao Xiaomin and Feng Zhi- 
jun introduced the concept of closed-loop supply chain management, and made some suggestions for electronics manufacturing implementation of closed-loop supply chain management in China [2]; Savaskan assumed that new products and remanufactured products for consumers were exactly the same, compared under different recycling channels of closed-loop supply chain efficiency, found that retailers recycling efficiency was higher than the third party recycling efficiency in closed-loop supply chain [3]; Based on the monopoly model, Ferrer studied the sales pricing decision of new products and remanufacturing products, and optimization problem of supply chain was discussed, he analyzed multiple periods of closed-loop supply chain optimization model, but the article also assumed that the new products and remanufactured products had the same quality and got a unified treatment in market [4]; Wang Yuyan studied the cooperating pricing strategy and non-cooperating pricing strategy in the closed-loop supply chain which composed of a manufacturer and a retailer [5]; Wang Wenbin and Da Qingli established the closed-loop supply chain model based on market segmentation, analyzed the rule about decision variables changing with exogenous variables, and compared the optimal decision under the closed-loop supply chain without recycling and manufacturing situation [6]; Li Xinjun studied the production optimization problem of two cycles under the condition of manufacturers and retailers, considering the remanufacturing cost was a random variable, quoting the concept of net consumer utility to analyze the market demand of new products and remanufacturing products and the corresponding mathematical model was established on this question [7]; Yi Yuyin studied the centrally coordinated system and three decentralized models with two dominant retailers, a centralized decision model was established and different dominant powers of closed-loop supply chain under decentralized decision-making game model, the retail price, the product return rate, and the profits of the channel members in the centrally coordinated and decentralized models were compared, finally he discussed the retailer leading closed-loop supply chain coordination mechanism [8].

Based on the analysis of existing study of closed-loop supply chain, our research considers that consumers have different preferences for new products and remanufactured products, based on this, we segment the product markets and set up a closed-loop supply chain decision-making model, our attention is focused on the market dominated by manufacturer, then the influence of consumer preference on sale price, wholesale price, recovery rate and the influence of profits is discussed.

\section{Model Description and Hypotheses}

\subsection{Model Description}

The object of our research is a manufacturer-leading closed-loop supply chain composed of one manufacturer, one retailer and the third-party recycler. As shown in Figure 1. In a single period, the third-party first recycles waste products from consumers at a certain price, and then sells all the recycled waste products to manufacturer, manufacturer pay recycler according to the recycling price to repurchase from those waste products and produce remanufactured products and new products at a certain cost then sell to retailer in different wholesale price. The retailer decides retail price based on the wholesale price of different products to maximize its profits.

Notation: variables, parameters, and demand function

$C_{n}$ : the unit cost of the new products,

$C_{r}$ : the unit cost of the remanufactured products, $C_{r}<C_{n}$,

$p_{n}$ : the unit sale price of the new products,

$p_{r}$ : the unit sale price of the remanufactured products,

$w$ : consumers' willingness to pay for new products,

$A$ : size of the potential market, constant every period,

$\omega_{n}$ : the unit wholesale price of the new products,

$\omega_{r}$ : the unit wholesale price of the remanufactured products,

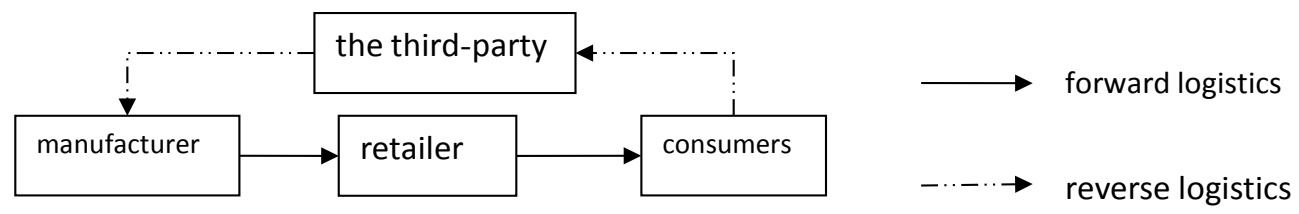

Figure 1. Closed-loop supply chain model. 
$\tau$ : the recovery rate of waste products, $0 \leq \tau \leq 1$,

$\delta$ : consumer preference coefficient, $0<\delta<1$,

$I$ : the third-party recycler's investment for recycling activities,

$a$ : the unit recycling price of the waste products,

$b$ : the unit repurchase price of the waste products, $b>a$,

$\pi_{i}$ : profit function, I $=\mathrm{M}, \mathrm{R}, 3 \mathrm{P}$.

\subsection{Hypothesis of the Model}

Hypothesis 1: Remanufactured products can meet the performance and quality of new products, but consumers still have different preference to them, this has led to consumers' different willingness to pay. Further, assume that w obeys a uniform distribution in $[0, A]$, so the price that consumers' willingness to pay for remanufactured products is $\delta w$.

Hypothesis 2: All of the recycled products can be remanufactured, $0 \leq \tau \leq 1$, the recovery rate $\tau$ is highly correlated to the recycler's investment for recycling activities. Refer to the advertising and operations research literature, we assume that $\tau=\sqrt{I / C}, C$ means scale parameter, $I=C \tau^{2}$ [9].

Hypothesis 3: Assume that every consumer only buys a product, the market is completely covered, and consumers can only choose to buy new products or remanufactured products.

Hypothesis 4: Assume that manufacturer is the market leader, who has absolute influence of channels for retailer and the third-party recycler.

Hypothesis 5: Assume that waste products market already exists, manufacturer and retailer are risk neutral, taking the maximum of the enterprises' benefits as the goal.

According to the model description and hypotheses, the profit function can be obtained.

The manufacturer's profit function is $\pi_{M}=\left(\omega_{n}-c_{n}\right) q_{n}+\left(\omega_{r}-c_{r}\right) q_{r}-b \tau\left(q_{n}+q_{r}\right)$.

The retailer's profit function is $\pi_{R}=\left(p_{n}-\omega_{n}\right) q_{n}+\left(p_{r}-\omega_{r}\right) q_{r}$.

The recycler's profit function is $\pi_{3 P}=\tau(b-a)\left(q_{n}+q_{r}\right)-C \tau^{2}$.

\section{The Establishment of the Model}

\subsection{Consumer Market Segmentation}

According to consumers' different willingness to pay and the psychological preference for new products and remanufactured products, we can segment market. Assume that the retail price of new products $p_{n}$ change within $[0, A]$, the retail price of remanufactured products pr change within $\left[0, p_{n}\right]$, so consumer's net utility obtained from purchasing a new product and a remanufactured product can be represented as $\mathrm{UN}=w-p_{n}, \mathrm{UR}=\delta w-p_{r}$ respectively. There are two extreme cases to consider.

The first is when consumers only buy new products. That is to say, consumers buy new products get the largest net utility, when $w-p_{n} \geq 0, \delta w-p_{r} \leq w-p_{n}$, so we can introduce that $w \geq \max \left\{p_{n},\left(p_{n}-p_{r}\right) /(1-\delta)\right\}$.

On the other extreme, consumers only buy remanufactured products. That is, consumers buy remanufactured products get the largest net utility, when $\delta w-p_{r} \geq 0, \delta w-p_{r} \geq w-p_{n}$, so we can introduce that $p_{r} / \delta \leq w \leq\left(p_{n}-p_{r}\right) /(1-\delta)$.

We have assumed that waste products market already exists, so we only research the situation that new products and remanufactured products sales are greater than 0 . According to two extreme cases discussed above, we can deduce that if $p_{r}<\delta p_{n}$, then $p_{r} / \delta<\left(p_{n}-p_{r}\right) /(1-\delta)$, remanufactured products sales is greater than 0 ; if $p_{r} \geq p_{n}-A(1-\delta)$, new products sales is greater than 0 . Therefore, when $p_{n}-A(1-\delta)<p_{r}<\delta p_{n}$, we can calculate the demand of new products $q_{n}$ and the demand of remanufactured products $q_{r}$ respectively.

$$
\begin{gathered}
q_{n}=A-\left(p_{n}-p_{r}\right) /(1-\delta) \\
q_{r}=\left(p_{n}-p_{r}\right) /(1-\delta)-p_{r} / \delta
\end{gathered}
$$

\subsection{Closed-Loop Supply Chain with the Third Party Collecting Model}

Manufacturer entrust a third party to recycle waste products, retailer is only responsible for selling products. 
Plug Equations (1) and (2) into profit function, we can get the profit function of the manufacturer, the retailer, the recycler.

$$
\begin{gathered}
\pi_{M}=\left(\omega_{n}-c_{n}\right)\left[A-\left(p_{n}-p_{r}\right) /(1-\delta)\right]+\left(\omega_{r}-c_{r}\right)\left[\left(p_{n}-p_{r}\right) /(1-\delta)-p_{r} / \delta\right]-b \tau\left(A-p_{r} / \delta\right) \\
\pi_{R}=\left(p_{n}-\omega_{n}\right)\left[A-\left(p_{n}-p_{r}\right) /(1-\delta)\right]+\left(p_{r}-\omega_{r}\right)\left[\left(p_{n}-p_{r}\right) /(1-\delta)-p_{r} / \delta\right] \\
\pi_{3 P}=\tau(b-a)\left(A-p_{r} / \delta\right)-C \tau^{2}
\end{gathered}
$$

Market dominated by manufacturer, so this is a Stackelberg game model led by the manufacturer. Supply chain members make the decision in this order: the manufacturer determines the wholesale price $\omega_{n}$, $\omega_{r}$, while the retailer determines the sale price $p_{n}, p_{r}$, and the third-party recycler determines the recovery rate $\tau$.

According to the backward induction, firstly, retailer decides the optimal sale price to maximize its profits. Take $\pi_{R}$ the derivative with respect to $p_{n}, p_{r}$, set the first-order derivative equal to zero, we can get the optimal sale prices are

$$
\begin{aligned}
& p_{n}=\frac{1}{2} A+\frac{1}{2} \omega_{n} \\
& p_{r}=\frac{1}{2} \delta A+\frac{1}{2} \omega_{r}
\end{aligned}
$$

The third-party recycler determines the recovery rate $\tau$, takes $\pi_{3 P}$ the derivative with respect to $\tau$, sets the first-order derivative equal to zero, the optimal recovery rate is

$$
\tau=\frac{b-a}{4 C}\left(A-\frac{\omega_{r}}{\delta}\right)
$$

Plug (6) (7) (8) into the manufacturer's profit function,

$$
\pi_{M}=\frac{1}{2}\left(\omega_{n}-c_{n}\right)\left[A-\frac{\omega_{n}-\omega_{r}}{1-\delta}\right]+\frac{1}{2}\left(\omega_{r}-c_{r}\right)\left[\frac{\omega_{n}-\omega_{r}}{1-\delta}-\frac{\omega_{r}}{\delta}\right]-\frac{b(b-a)}{8 C}\left(A-\frac{\omega_{r}}{\delta}\right)^{2}
$$

Manufacturer determines the wholesale price $\omega_{n}, \omega_{r}$ to maximize its profits, take $\pi_{M}$ the derivative with respect to $\omega_{n}, \omega_{r}$, set the first-order derivative equal to zero, the optimal wholesale prices are

$$
\begin{gathered}
\omega_{n}=\frac{4 C \delta\left(A+c_{n}\right)+b(b-a)\left(A \delta+A+c_{n}-c_{r}\right)}{2[b(b-a)+4 C \delta]} \\
\omega_{r}=\frac{2 C \delta\left(A \delta+c_{r}\right)+b(b-a) A \delta}{b(b-a)+4 C \delta}
\end{gathered}
$$

Then, plug $\omega_{n}, \omega_{r}$ into $p_{n}, p_{r}, \tau$

$$
\begin{gathered}
p_{n}=\frac{4 C \delta\left(3 A+c_{n}\right)+b(b-a)\left(A \delta+3 A+c_{n}-c_{r}\right)}{4[b(b-a)+4 C \delta]} \\
p_{r}=\frac{C \delta\left(3 A \delta+c_{r}\right)+b(b-a) A \delta}{b(b-a)+4 C \delta} \\
\tau=\frac{\left(A \delta-c_{r}\right)(b-a)}{2[b(b-a)+4 C \delta]}
\end{gathered}
$$

At last, we can know the demand of new products and remanufactured products

$$
q_{n}=\frac{A(1-\delta)+c_{r}-c_{n}}{4(1-\delta)}
$$




$$
\begin{gathered}
q_{r}=\frac{4 C \delta\left(\delta c_{n}-c_{r}\right)+\delta b(b-a)\left(A \delta-A+c_{n}-c_{r}\right)}{4[b(b-a)+4 C \delta]} \\
q=\frac{C\left(A \delta-c_{r}\right)}{b(b-a)+4 C \delta}
\end{gathered}
$$

\section{Numerical Example Analysis and Conclusions}

Finally, a numerical example is presented to analyze this closed-loop supply chain system. Assignments for the relevant parameters are $c_{n}=15, c_{r}=5, C=1000, A=200, a=3, b=4.5$. Through the market segmentation, we can calculate when $\delta$ is within [0.422, 0.950], new products and remanufactured products' demands are greater than 0 . Next we take $\delta=0.5,0.6,0.7,0.8,0.9$ to observe the change trends of decision variables, sales, profits change with consumer preference. By calculation the upper situation, we have come to the following conclusions.

Conclusion 1: As shown in Figure 2 and Figure 3, the wholesale price and sale price of remanufactured products increase with consumer preference significantly, while the effect that increases in consumer preference for wholesale price and sale price of new products is not obvious even slightly lower.

This indicates that because the remanufactured products have the same quality with new products, as long as the consumers gradually recognize this point, the price of remanufactured products will be more and more close to the price of new products.

Conclusion 2: As shown in Figure 4, the total sales volume increases with the increase of consumer preference slightly, but the sales of new products and the sales of remanufactured products change differently with consumer preference, $q_{n}$ reduces with the increase of consumer psychology preference significantly, while $q_{r}$ increases with consumer preference obviously.

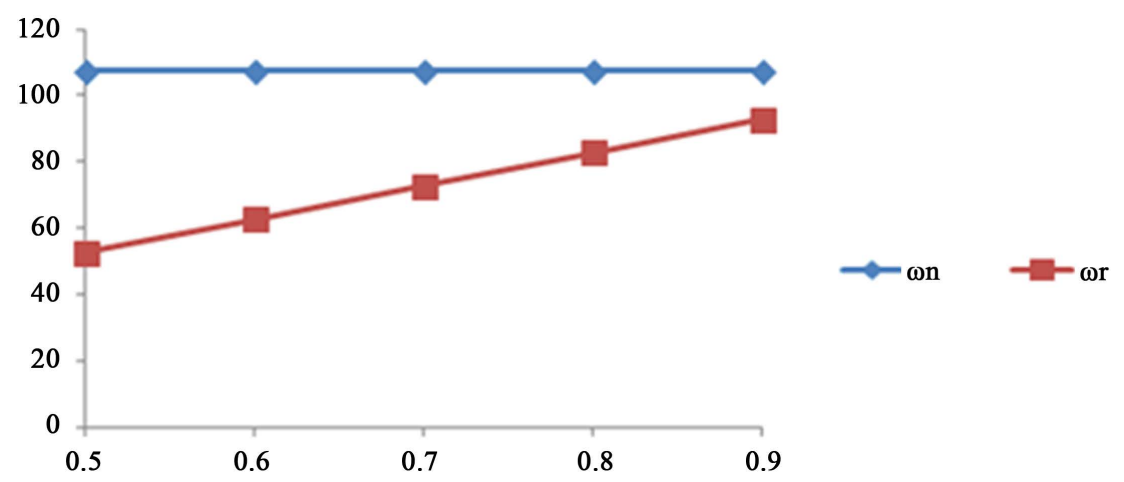

Figure 2. Wholesale prices change with consumer preference.

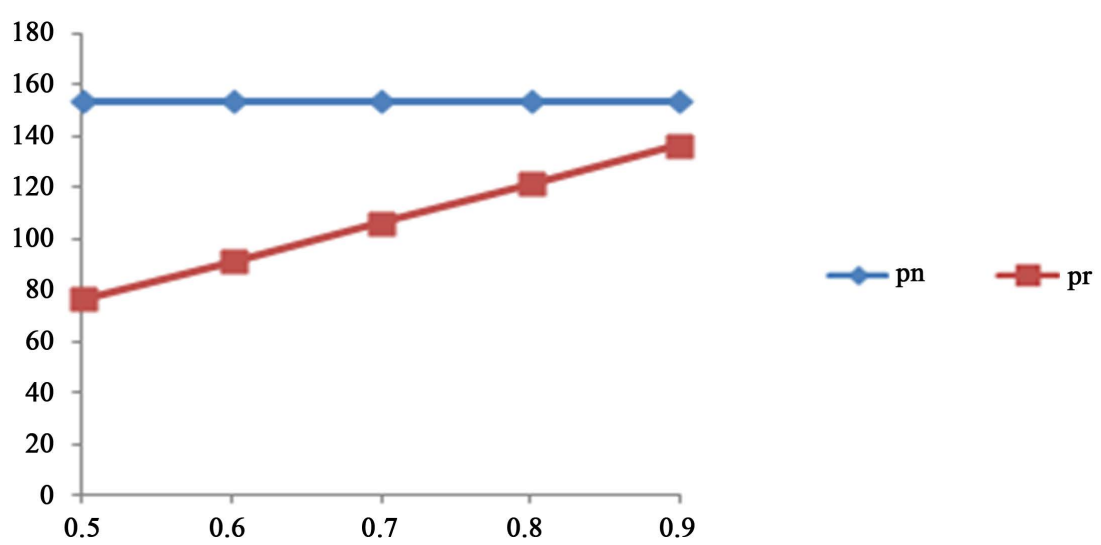

Figure 3. Sale prices change with consumer preference. 
This shows that as consumers get more and more recognition to the quality of the remanufactured products, and its price is lower than new product, so more and more people will choose to buy remanufactured products, which leads to reduce the purchase of new products, but on the whole, total sales still increases with the increase of consumer preference.

Conclusion 3: The recovery rate of waste products increases as consumer preference. Figure 5 indicates that consumers' identity improvement caused the increase of remanufactured products demand, so the third-party needs to strengthen the recycling to satisfy the demand of the market.

Conclusion 4: As the consumer preference increases, the profits of different parties are increased, but the increasing extent is different. As shown in Figure 6 and Figure 7, we can see that consumers' identity improvement increases the entire supply chain system profits, and growth amplitude is bigger and bigger, which reflects the benefits of implementing closed-loop supply chain members to all parties concerned.

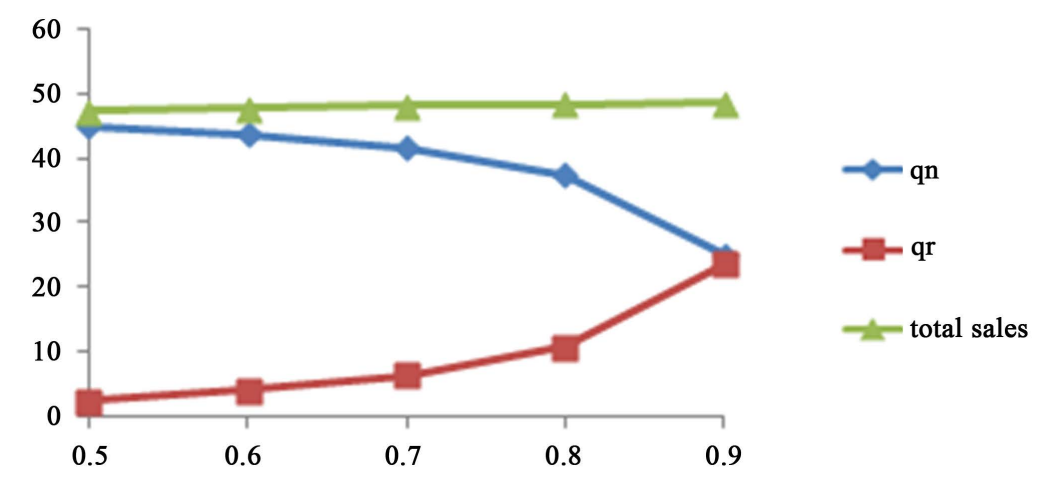

Figure 4. Sales change with consumer preference.

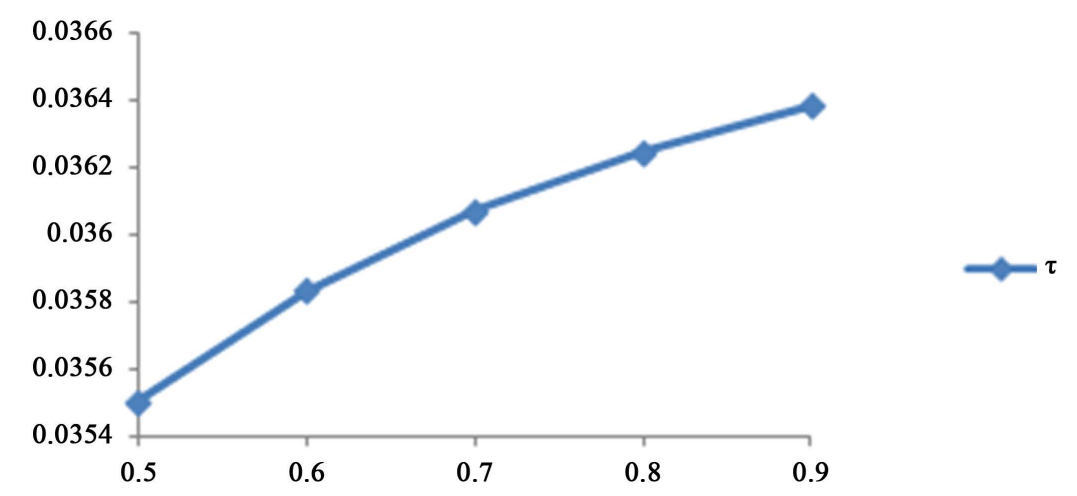

Figure 5. Recovery rate change with consumer preference.

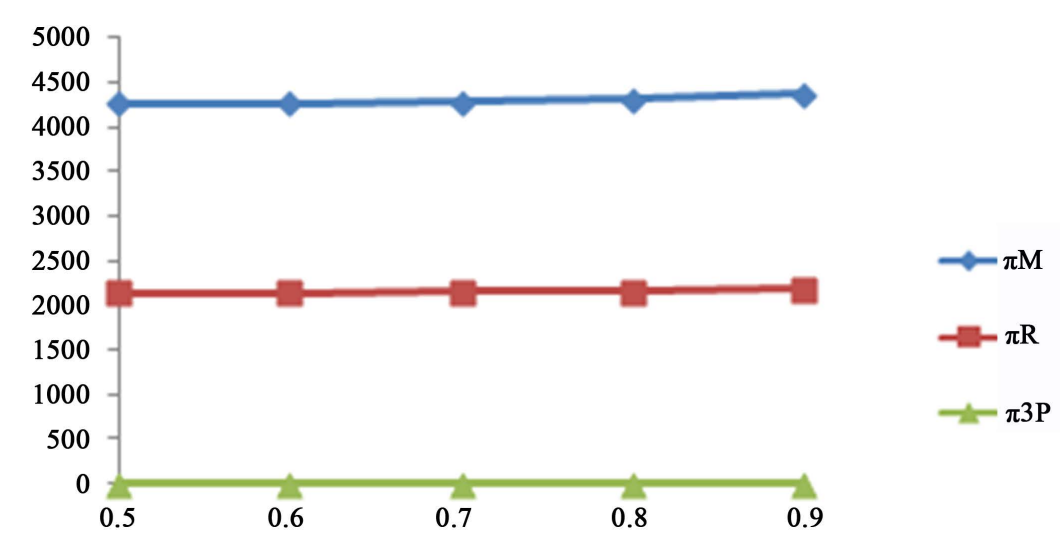

Figure 6. Profits change with consumer preference. 


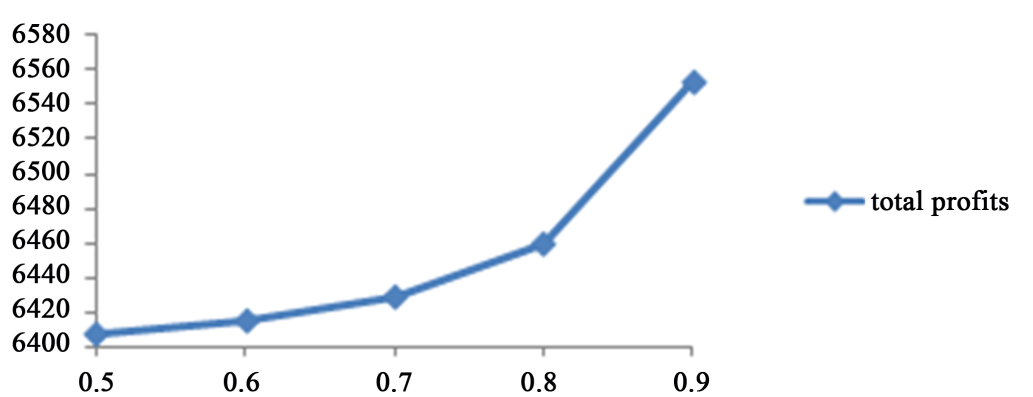

Figure 7. Total profits change with consumer preference.

\section{Future Research}

Based on the assumption that consumer conveys different preference for new products and remanufactured products, we segment markets and determine the market demand of different products. Then, we set up a closedloop supply chain with the third party collecting model and obtain the optimal solutions; the influence of consumer preference on sale price, wholesale price, recovery rate and profits is discussed; and we draw a series of corresponding conclusions.

In the finality, we propose some directions for further research, such as considering the comparison under different market structures, including the power structure and topology structure; comparing the recovery efficiency under different recycling channels; considering not completely covered market cases; broadening the research hypotheses, and so on.

\section{References}

[1] Guide, V.D.R. and Wassenhove, L.N.V. (2003) Business Aspects of Closed-Loop Supply Chains. Carnegie Mellon University Press, Pittsburgh.

[2] Zhao, X.M. and Feng, Z.J. (2004) Closed-Loop Supply Chains Management-Managerial Innovation on Meeting WEEE EU Directive in Our Electronic Industries. China Industrial Economics, 197, 48-55.

[3] Savaskan, R.C., Bhattacharya, S. and Van Wassenhove, L.N. (2004) Closed-Loop Supply Chain Models with Product Remanufacturing. Management Science, 50, 239-252. http://dx.doi.org/10.1287/mnsc.1030.0186

[4] Ferrer, G. and Swaminathan, J.M. (2006) Managing New and Remanufactured Products. Management Science, 52, 15-26. http://dx.doi.org/10.1287/mnsc.1050.0465

[5] Wang, Y.Y. and Li, B.Y. (2006) The Research on Two Price Decision Model of Closed-loop Supply Chain. The Journal of Forecast, 25, 70-73.

[6] Wang, W.B. and Da, Q.L. (2009) Production and Pricing Strategy of Closed-Loop Supply Chain with Consumer Segmentation. Control and Decision, 24, 675-679.

[7] Li, X.J. (2007) Monopoly Production Optimization Model for Remanufacturing Cost with Statistic Distribution and Market Segmentation. Chinese Journal of Management Science, 15, 72-77.

[8] Yi, Y.Y. (2010) Study on Closed-Loop Supply Chain Models under Different Market Power. Systems EngineeringTheory Methodology Application, 4, 389-396.

[9] Savaskan, R.C. and Van Wassenhove, L.N. (2006) Reverse Channel Design: The Case of Competing Retailers. Management Science, 52, 1-14. http://dx.doi.org/10.1287/mnsc.1050.0454 